\title{
Controlled study of 24-hour ambulatory electrocardiographic monitoring in patients with transient neurological symptoms
}

\author{
LINDA M LUXON, ALEC CROWTHER, MICHAEL JG HARRISON, AND D \\ JOHN COLTART
}

From the Department of Neurological Studies, The Middlesex Hospital, and Department of Cardiology, St Thomas' Hospital, London

SUMMARY Sixty unselected patients with transient neurological symptoms underwent 24-hour ambulatory electrocardiographic (ECG) monitoring. Haemodynamically significant arrhythmias were observed in $32 \%$ of patients, but in only $3 \%$ of an age- and sex-matched control group. Nine patients received specific anti-arrhythmic therapy, which resulted in marked symptomatic improvement in each case. It is argued that 24-hour ECG monitoring is a valuable diagnostic tool in the investigation of transient neurological symptoms.

The interpretation of transient neurological disturbances is often difficult. Syncope, epilepsy, vestibular episodes, and ischaemic attacks all need to be considered. Patients often give only a vague account of an attack and frequently the diagnosis is still in doubt after neurological and cardiological examination.

The possibility that such symptoms are caused by an intermittent cardiac arrhythmia ${ }^{1-6}$ is difficult to exclude, unless by chance an attack is witnessed. The present study was therefore devised to assess the value of 24-hour ambulatory ECG monitoring in a wide spectrum of patients with episodic neurological symptoms. Results of recent studies ${ }^{7}$ have shown a high incidence of ECG abnormalities on prolonged monitoring of normal adults and so it was considered essential to study a control population.

\section{Patient selection and recording technique}

Sixty consecutive patients, referred to a neurological department for assessment of transient cerebral symptoms, were studied. The criteria for selection depended solely on their willingness

Address for reprint requests: $\mathrm{Dr}$ Linda $M$ Luxon, The National Hospital for Nervous Diseases, Queen Square, London WC1N 3BG.

Accepted 25 June 1979 to cooperate in the study and the transient nature of any symptoms. In addition to routine clinical assessment and neurological investigations, a resting 12-lead ECG and an ambulatory 24-hour ECG record were obtained for each patient. Twenty-four hour ECG records were also obtained for age and sex-matched control subjects who were chosen from volunteers among the employees and pensioners of a large London department store. Age matching was to within one year of each patient. The sole reason for exclusion of a volunteer was a past history of transient neurological symptoms.

An Oxford Medilog cassette recorder with a single channel for ECG was used. The electrodes were applied in the VI and V5 positions to give an ECG configuration similar to V5. The recordings were obtained for 24 hours with the patients performing their daily activities. In the studied group 17 were inpatients, but were fully ambulant during the day. The recording was repeated if an incomplete (less than 24 hours) record was obtained or if, on subsequent analysis, the trace was deemed to be of poor quality. The tapes were analysed with the aid of a purpose-built arrhythmia detector (Oxford Instruments Ltd). This unit incorporates a memory oscilloscope which allows any detected abnormality to be observed in real time and written out on standard ECG paper for formal analysis. The tapes were 
processed by the same experienced technician (AC) in a blind fashion and were reviewed by two observers (LML and DJC). All arrythmias were therefore recorded, but no quantification of their frequency was made.

For the purpose of this study haemodynamically significant arrhythmias were defined as sinus arrest greater than 1.6 times the preceding $R-R$ interval, supraventricular or junctional tachycardia or both, atrial fibrillation with block, rapid atrial fibrillation of greater than 110 beats per minute, complete atrioventricular dissociation, runs of coupled ventricular ectopic beats, salvoes of ventricular ectopic beats, and ventricular tachycardia.

It has been established that both atrial and ventricular extrasystoles can produce a marked fall in peak blood flow $^{8}$ and may therefore give rise to transient cerebral symptoms. However, as it was impossible to quantitate the frequency of single extrasystoles with the playback equipment available and as clear criteria for normality, with respect to extrasystoles, have not been established, single extrasystoles were not considered "significant".

\section{Results}

There were 27 females and 33 males in both the symptomatic and control groups. The female patient age range was 16-79 years (mean 60.5 years), compared with 16-80 years (mean 58.9 years) in the control group and for males 22-73 years (mean 55.2 years) and 23-74 years (mean $55 \cdot 1$ years) respectively.

The clinical diagnosis in the 60 neurological patients was noted. In 12 the physician diagnosed epilepsy, since jactitation, incontinence, or self- injury had occurred. In 27 the attacks appeared syncopal. Ten further patients described light headedness or vertigo or both, and the diagnosis was, therefore, less certain, but anxiety states were considered in three and mild vertebrobasilar insufficiency in seven. The remaining 11 patients had transient ischaemic attacks; episodic focal neurological deficits, without alteration of consciousness.

An arrhythmia was found in $82 \%$ of patients on prollonged monitoring, compared with $43 \%$ of the control group (table 1). Significant arrhythmias occurred 10 times more commonly in the patients and were most frequently supraventricular in origin. In the control group the significant arrhythmias that occurred were both ventricular in origin. However, a single arrhythmia was unccmmon and in $70 \%$ of patients with a significant abnormality, two or more arrhythmias were demonstrated.

A past history of ischaemic heart disease was positively correlated with the occurrence of a significant arrhythmia. No such correlation could be established with other clinical features (chest pain or palpitations during an attack, added heart sounds, cardiac murmurs, bruits, or absent peripheral pulses).

Routine ECGs revealed an arrhythmia in seven patients (two atrial fibrillation, four sinus bradycardia, one sinus arrest), all of whom were found to have a significant arrhythmia on prolonged monitoring. A further $37 \%$ of patients showed conduction defects or repolarisation changes on routine ECGs but these changes were not significantly related (chi square test) to the cccurrence of an arrhythmia and would not normally indicate the need for further cardiological investigation.

Table 1 Arrhythmias detected by ambulatory 24-Hour ECG monitoring

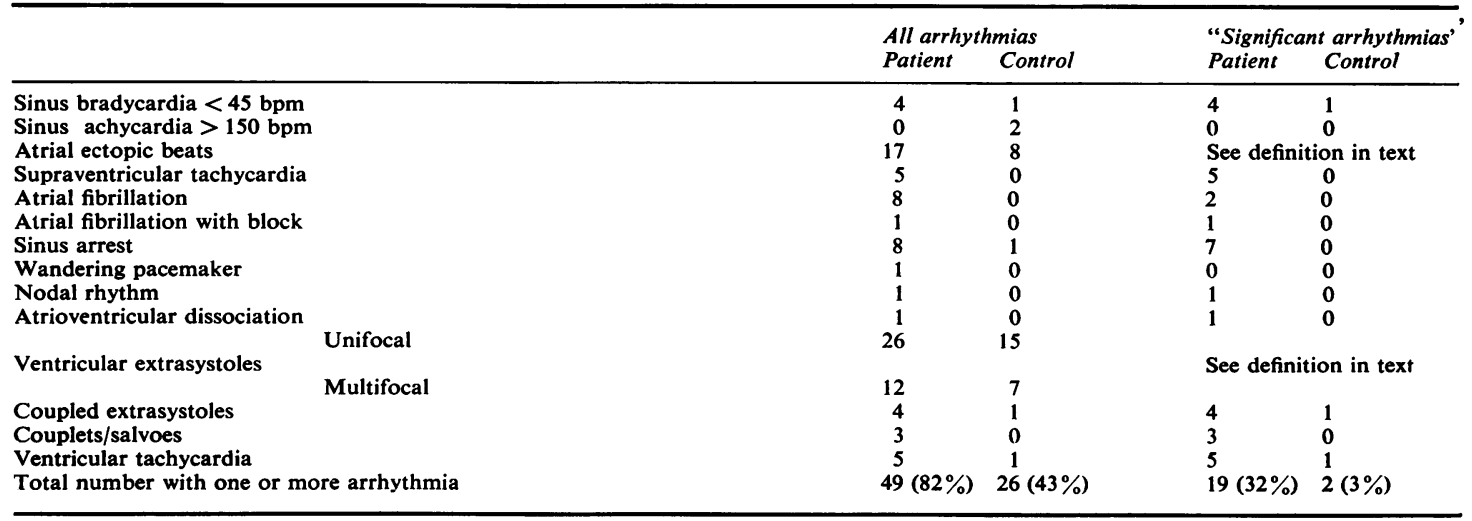


Nine patients with significant arrhythmias received specific anti-arrhythmic treatment (table 2). Three patients (two with syncope and one with epilepsy) required pacemaker insertion and were asymptomatic during a six-month followup period. Six patients received medical treatment (propranolol, disopyramide, procainamide, or mexilitene) and two were rendered asymptomatic, while there was improvement in the frequency and severity of symptoms in the remaining four patients.

One patient with syncope, thought to be secondary to sinus arrest, improved spontaneously. The only patient with an arrhythmia in the "dizzy" group was considered to be suffering from vertebrobasilar insufficiency. He was treated with aspirin and improved symptomatically. In the epilepsy group, two patients with significant arrhythmias underwent electrophysiological studies, confirming the presence of sinus node disease. Neither patient received specific therapy, as one patient improved spontaneously and the other experienced very infrequent symptoms. Both remain under review. The remaining patient with epilepsy and an arrhythmia died of carcinoma of the stomach shortly after inclusion in this study.

In the group of transient ischaemic attacks with arrhythmias one patient improved after carotid endarterectomy, two improved on anticoagulants and one on aspirin, and the remaining patient returned to his home abroad and was lost to follow-up.

\section{Discussion}

An arrhythmia was found in $82 \%$ of patients on prolonged monitoring as compared to $43 \%$ of the control group. This high incidence corresponds well with the results of other workers. ${ }^{4}{ }^{4} 10$ Prolonged cardiac monitoring has resulted in the necessity for reappraisal of the "normal" heart rhythm $^{7}$ and therefore, for the purposes of this study an attempt was made to define "significant arrhythmias" which would be clearly accepted as haemodynamically abnormal. On the basis of

Table 2 Distribution of significant and specific therapy in clinical subgroups

\begin{tabular}{llll}
\hline Clinical subgroup & Number & $\begin{array}{l}\text { Significant } \\
\text { arrhythmias }\end{array}$ & $\begin{array}{l}\text { Anti-arrhythmic } \\
\text { therapy }\end{array}$ \\
\hline Dizziness \pm vertigo & 10 & 1 & 0 \\
Syncope \pm dizziness & 27 & 9 & 8 \\
$\begin{array}{l}\text { Epilepsy } \\
\text { Transient ischaemic }\end{array}$ & 12 & 4 & 1 \\
attacks & 11 & 5 & 0 \\
\hline
\end{tabular}

this definition, $32 \%$ of the patient group were found to have significant arrhythmias in comparison to $3 \%$ of the controls. ${ }^{5}$ Arrhythmias in patients with transient cerebral symptoms tended to be supraventricular in origin, ${ }^{5}{ }^{11}$ while the two control subjects with a significant abnormality both showed a ventricular arrhythmia. Two control subjects were noted to have sinus tachycardia, 150 beats per minute, but as heavy manual work was being undertaken at the time, the heart rate was not felt to be inappropriate.

Reviewing the histories and examinations of the patient group, the only positive relationship established was between a past history of heart disease and the occurrence of a significant arrhythmia. In contrast to other studies, the symptoms of chest pain or palpitations or both ${ }^{5}$ or indeed the presence of physical signs of cardiovascular diseases, ${ }^{9}$ were unhelpful in suggesting an underlying arrhythmia.

Routine ECGs revealed an arrhythmia in seven patients and all of these subsequently proved to have significant abnormalities on prolonged monitoring. However, it should be emphasised that nine patients with significant arrhythmias had normal ECGs, and a further three had only conduction or repolarisation changes, which would not normally have raised the suspicion of an underlying arrhythmia. ${ }^{259}$ Indeed, in the present study there was no relationship (chi square test) between conduction abnormalities or repolarisation changes and the occurrences of an arrthymia upon monitoring.

Considering individual clinical groups, the numbers were small and dogmatic statements cannot be made, although certain points do emerge. Significant arrhythmias occurred most rarely in patients with dizziness or vertigo or both, compared with an incidence of $33 \%$ in patients presenting with syncope. This disparity is difficult to explain as "dizziness" and syncope may be different degrees of the same underlying pathological process-that is, generalised cerebral ischaemia. Nonetheless, it would appear that in neurological practice an underlying arrhythmia should be more strongly suspected in patients with syncopal attacks. Reed $e t a^{12}$ reported that the majority of 290 patients requiring pacemaker insertion for cardiac arrhythmias or conduction abnormalities, presented with syncope.

Thirty-three per cent of patients with epileptiform attacks were also found to have significant arrhythmias. Phizackerley et $a l^{13}$ first reported sino-auricular heat block as a visceral manifestation of epilepsy. Conversely, Schott et al $^{3}$ reported that $20 \%$ of patients referred to a neurological 
department with possible idiopathic epilepsy were subsequently found to have cardiac arrhythmias that caused, or significantly contributed to, their symptoms. Three patients with epilepsy in this study were proven electrophysiologically to have sinus node disease and in one of these the symptoms were abolished completely by pacemaker insertion. It would therefore appear that the epileptic attacks were precipitated by the arrhythmia, rather than vice versa. In order to establish the exact sequence of events it would be necessary to obtain simultaneous EEG and ECG monitoring in some patients. This latter procedure might also help to elucidate the role of cardiac arrhythmias in sudden death among epileptics. ${ }^{14}$ The remaining patient with epilepsy died from carcinomatosis without full investigation. It is, therefore, highly probable that his arrhythmia was incidental and his epilepsy secondary to cerebral metastases. Excluding this patient, the number of epileptics with significant arrhythmias in this study correlates well with the findings of Schott et $a l^{3}$

Most transient ischaemic attacks are believed to be caused by microembolism, but haemodynamic factors are occasionally responsible and the possibility of an intermittent arrhythmia should be considered. The focal symptoms of transient ischaemic attacks are unlikely to be the result of a fall in cardiac output produced by an arrhythmia unless there is coexistent narrowing of a cerebral vessel. ${ }^{11-12}$ The first successful carotid endarterectomy was carried out on a patient who experienced monocular blindness or a hemiparesis or both every time she had a run of paroxysmal tachycardia. ${ }^{15}$ After successful repair of the carotid stenosis, the arrhythmia persisted but no longer caused transient cerebral symptoms. Focal symptoms were, in fact, rare in the large series of patients experiencing symptomatic arrhythmias described by Reed et al. ${ }^{12}$ Arrhythmias in patients with cerebrovascular disease may merely be a manifestation of their more widespread atherosclerosis and, therefore, the finding of an arrhythmia on prolonged cardiac monitoring ( $45 \%$ in this study) needs careful assessment.

Despite the high incidence of arrhythmias we observed only one patient suffered symptoms at the time of a recorded arrhythmia. If symptoms are infrequent, the likelihood of their occurring during a 24-hour recording is remote and it may be necessary to monitor for longer periods. Only one patient in the present series underwent repeated monitoring (96 hours) before an arrhythmia was detected. In this instance prolonged neurological investigations had failed to reveal a cause for recurrent syncopal attacks. The presence of symptoms and arrhythmias on 24 hour monitoring does not prove a cause and effect relationship unless they occur simultaneously. However, if asymptomatic arrhythmias are detected on ambulatory monitoring and full neurological investigation is negative, there may be indication for further electrophysiological cardiac investigation or a trial of anti-arrhythmic therapy or both. In the present study six such patients underwent electrophysiological studies and in five a sinus node abnormality was established. Conversely, 24-hour monitoring may yield valuable negative information if a patient's typical symptoms occur in the absence of a cardiac arrhythmia.

In three patients with electrophysiologically proven sinus node disease the abnormality was considered sufficiently severe to warrant pacemaker insertion. As the course of this disorder may be benign, a period of observation is in progress in two further patients. All six patients with significant arrhythmias, who were treated with anti-arrhythmic drugs, showed a good symptomatic response.

We conclude that ambulatory 24-hour monitoring can be of great value in the assessment of patients with episodic neurological symptoms.

We should like to thank Dr C J Earl, Dr W M Dixon, and Dr S Joseph for their help and encouragement, and to acknowledge the invaluable secretarial assistance of Mrs F Baile and Miss S Munson.

\section{References}

1 Editorial. In the heart or in the head. $\mathrm{Br}$ Med J 1976; 1:1158.

2 McAllen PM, Marshall J. Cardiac dysrhythmia and transient cerebral ischaemic attacks. Lancet $1973 ; 1: 1212-14$.

3 Schott GD, McLeod AA, Jewitt DE. Cardiac arrhythmias that masquerade as epilepsy. $\mathrm{Br}$ Med J 1977; 1:1454-7.

4 Abdon NJ, Malmcrona R. High pacemaker implantation rate following "Cardiogenic Neurology". Acta Med Scand 1975; 198:455-61.

5 Goldberg AD, Raftery EB, Cashman PMM. Ambulatory electrocardiographic records in patients with transient cerebral attacks of palpitation. Br Med J 1975; 4:569-71.

6 Tzivoni D, Stern S. Pacemaker implantation based on ambulatory ECG monitoring in patients with cerebral symptoms. Chest 1975; 67:274-8.

7 Brodsky M, Wu D, Denes P, Kanakis C, Rosen KM. Arrhythmia documentation by 24hour continuance of electrocardiographic 
Controlled study of 24-hour ambulatory electrocardiographic monitoring in patients with transient 41

monitoring in 50 male medical students without apparent heart disease. Am J Cardiol 1977; 39: 390-5.

8 Benchimol A, Maroko P, Gartlan J. Continuous measurement of arterial flow in man during atrial and ventricular arrhythmias. $\mathrm{Am}$ J Med 1969; 46:52-63.

9 Levin EB. Use of the holter electrocardiographic monitor in the diagnosis of transient ischemic attacks. J Am Geriatr Soc 1976; 24:516-21.

10 Hinckle LE, Carver ST, Stevens M. Frequency of asymptomatic disturbance of cardiac rhythm and conduction in middle-aged men. $A m ~ J$ Cardiol 1969; 24:629-50.

11 Walter PF, Reid SD, Kass Wenger N. Transient cerebral ischemia due to arrhythmia. Ann Intern Med 1970; 72: 471-4.

12 Reed RL, Siekert RG, Meredith JJ. Rarity of transient focal cerebral ischemia in cardiac dysrhythmia. JAMA 1973; 223:893-5.

13 Phizackerley PJR, Poole EW, Whitty CWM. Sino-auricular heart block as an epileptic manifestation. Epilepsia 1954; 3:89-96.

14 Hirsch CS, Martin DL. Unexpected death in young epileptics. Neurology (Minneap) 1971; 21:682-90.

15 Eastcott HHG, Pickering GW, Rob CG. Reconstruction of internal carotid artery in a patient with intermittent attacks of hemiplegia. Lancet 1954; 2:994-6. 